\title{
Significance of Neutrino Environments on Neutrino-Induced Fission and Neutron-Induced Fission Processes
}

Muhammad Ali

Awan Clinic Faisalabad, Pakistan

Email: alinaeem023@hotmail.com; m.alinaeem23@gmail.com

Orcid ID: 0000-0002-5760-912X

\begin{abstract}
This paper presents Mathematical Formulation for Neutrino-Fission of a heavy nuclei in Neutrino-environments. It has been proposed that neutrino fission is a dynamic twoprocess reaction, where more than one neutrino can be captured by target nucleus. A quantity named Neutrino-Zeta $\zeta$ with units of $\mathrm{eV}^{-1}$ has been defined to represent Neutrino-environments. Neutrino-zeta with assumed excited energy states for heavy nuclei $\mathrm{A} \sim 230-270$ is estimated to be $\zeta \approx 0.0212 \mathrm{MeV}^{-1}$, with the probability of fission Barrier energy state $P\left(E_{X, f}\right)=0.169064014$, with $E_{X, f}=6 \mathrm{MeV} . P\left(E_{X, f}\right)=$ 0.222664 is highest at $\zeta=0.1 \mathrm{MeV}^{-1}$. It has been shown that probabilities of certain excitation energy states and their corresponding decay reactions at those excitation energies can be equal for two neutrino-zetas. Neutron induced fission for different neutrinos hypothesize that probability of symmetric fission channels is higher at low neutrino-zetas, which can be tested experimentally.
\end{abstract}

\section{Keywords}

Neutrino-Induced Fission; Neutron-Induced Fission; Neutrino-Environments; Neutrino-Zeta; Fission Fragments; Supernova

\section{Introduction}

Neutrino-Induced Fission of heavy nuclei is a well-studied reaction, especially at Supernova ${ }^{1,2,3,4}$ sites and r-processes, where neutrino-fluxes are high of the order $10^{42}$ $\mathrm{cm}^{2} / \mathrm{s}$. It has been realised that neutrinos at high energies leave heavy nuclei at higher excitation energies, making them vulnerable for fission decay ${ }^{4}, 5$. Furthermore, significant research has been aimed to study neutrino interactions with matter ${ }^{6}$. Neutrino interactions with radionuclides have been proposed and their reaction crosssections have been theorised and studied ${ }^{7,8}$. This paper aims to study neutrino fission of a target nuclei in neutrino environments, where more than one neutrino can be captured by the target nuclei. The two-process reaction for neutrino fission has been presented in section 2. The exact number of neutrino captures is an arduous technological challenge, so Mathematical formulation using statistical mechanics has been employed in section 3 to derive Probability Distribution $P\left(E_{X, i}\right)$ of excited energy states $E_{X, i}$ of a target nucleus. A quantity named neutrino-zeta $\zeta$ with the units of $\mathrm{eV}^{-1}$ has been defined, which governs the probability distribution of these excited energy 
states. The probability distributions are exponential functions. In section 4 , the neutrino-zeta $\zeta$ has been estimated in Supernova for r-process sites, using assumed number and energy states of target nucleus and second order Maclaurin series for exponential functions. Quadratic formula for neutrino-zeta has been derived. The estimated value of neutrino-zeta at supernova is approximately $\zeta=0.0212 \mathrm{MeV}^{-1}$. Based on this neutrino-zeta, the probability distribution for an arbitrary heavy nuclei A 230-270 has been evaluated. In section 4 effects of neutrino zeta on certain energy states have been studied. Section 5 presents neutron induced fission reaction in neutrino-environments. The probability distribution of excitation energy states $P\left(E_{v}^{*}\right)$ by neutrino(s) interactions have been studied for different neutrino-zeta. Lastly, it has been hypothesized that the probabilities of symmetric fission channels are higher for lower neutrino-zeta values, which can be experimentally tested for different neutrinozeta values.

\section{Neutrino-Induced Fission}

This section presents the neutrino-induced fission reaction of heavy nuclei. The presented reaction comprises of two processes 1 and 2 as shown in equation 1.

$\sum a_{f} v_{f}+X \stackrel{1}{\rightarrow}[X]^{*}+\sum b_{f} v_{f} \stackrel{2}{\rightarrow}[Y]^{*}+[Z]^{*}+\sum_{i}^{m}\left[n_{i}\right]^{*}+\sum c_{f} v_{f}$

$\sum a_{f} v_{f}$ represents neutron number density, with $a_{f}$ denotes the number of neutrinos and f represents the neutrino flavor. Therefore, neutrino number density is the sum of all three neutrino flavors. $X$ represents the target heavy nucleus.

\subsection{Process 1}

Process 1 is the nucleus excitation process, which is induced by neutrinos. The reaction is given by equation 2

$\sum a_{f} v_{f}+X \stackrel{1}{\rightarrow}[X]^{*}+\sum b_{f} v_{f}$

$[X]^{*}$ represents the excited nucleus, which is achieved by capturing neutrinos given by $[X]^{*}=X+\sum x_{f} v_{f}$ where $x_{f}$ is the number of captured neutrinos by the target nucleus. Therefore,

$\sum x_{f} v_{f}=\sum a_{f} v_{f}-\sum b_{f} v_{f}$

$\sum b_{f} v_{f}$ is the number density of uncaptured neutrinos. Process 1 is the dynamic process, which is the combination of two types of processes at any instance i.e. neutrino scattering and neutrino capture. However, the condition $\sum a_{f} v_{f} \geq \sum b_{f} v_{f}$ is always met. 


\section{Neutrino Scattering $x_{f}=0$}

If the number of neutrinos in process 1 are equal i.e. $a_{f}=b_{f}$, it represents neutrino scattering. The type of neutrino scattering can be coherent elastic scattering ${ }^{8,9}, 10$, incoherent elastic scattering ${ }^{11}$ or the combination of both. The kinetic energy and momentum of neutrinos are changed. The kinetic energy and momentum of target nucleus changes too, such that the total momentum and energy is conserved.

\section{Neutrino Capture $x_{f}>0$}

If the number of neutrinos after process 1 is unequal i.e. $a_{f}>b_{f}, x_{f}$ neutrinos are captured by the nucleus. The resulting captures, which can be one or more than one, excites the target nucleus. Furthermore, captured neutrinos can be of any flavor, depending on the microscopic capture cross-sections of different flavors with the target nucleus and their chemical potentials. In addition, these cross-sections also depend on the type of current reaction (Charged-current or Neutral-current). The charged current neutrinos reactions with heavy nuclei shows higher cross-sections, however neutral current reactions can be induced by $v_{\mu, \tau}$ at higher average energies of $\left\langle e_{v}>\sim 20-\right.$ $25 \mathrm{MeV}$ for example in a core bounce supernova explosion ${ }^{1}$. The excitation energy state of the target nucleus can lead to different decay modes, depending on the excitation of the target nucleus. The higher excitation energy states can leave the target nucleus vulnerable for fission ${ }^{3,4}$.

\subsection{Process 2}

Assuming, the target nucleus has attained minimum excitation energy state equal to the fission barrier i.e. $E_{X, f}$, the nucleus irretrievably decays into fission fragments with the possible release of neutrons. The fission reaction is given by equation 4 .

$[X]^{*}+\sum b_{f} v_{f} \stackrel{2}{\rightarrow}[Y]^{*}+[Z]^{*}+\sum_{i}^{m}\left[n_{i}\right]^{*}+\sum c_{f} v_{f}$

$[Y]^{*}$ and $[Z]^{*}$ are the fission fragments which can be in the excited state because of the presence of captured neutrinos, therefore $[Y]^{*}=Y+\sum y_{f} v_{f}$ and $[Z]^{*}=Z+$ $\sum z_{f} v_{f}$, with $y_{f}$ and $z_{f}$ are the number of neutrinos present in the daughter nuclei $Y$ and $\mathrm{Z}$ respectively. In addition, the possibly released neutrons can be in the excited state $\left[n_{i}\right]^{*}$ with the presence of neutrinos, where $i=0,1,2, \ldots$ representing the number of neutrons released, with $m$ are the maximum number of neutrons released. Therefore, $\left[n_{i}\right]^{*}=n_{i}+\sum d_{i, f} v_{f}$ with $d_{i, f}$ are the number of neutrinos presents in the individual released neutrons. $\sum c_{f} v_{f}$ is the remaining neutrino number density.

\subsection{Conservation Laws for the reaction}

In the reaction in equation 1, the conservation laws for the neutrinos hold. Firstly, the number density of neutrinos is conserved, given by equation 5 .

$\sum a_{f} v_{f}=\sum b_{f} v_{f}+\sum x_{f} v_{f}=\sum y_{f} v_{f}+\sum z_{f} v_{f}+\sum d_{i, f} v_{f}+\sum c_{f} v_{f}$ 
It is speculated neutrino oscillations may occur with the interaction of matter ${ }^{12}$, nonetheless number of neutrinos remain the same. In addition to that, the conservation of mass and subsequently the energy of the reaction is also conserved, given by equation 6 and 7 for Process 1 and 2 respectively.

$$
\begin{aligned}
& \sum m_{v_{f}} \cdot v_{a, f}+m_{X}(X)=\sum m_{v_{f}} \cdot v_{x, f}+\sum m_{v_{f}} \cdot v_{b, f}+m_{X}(X) \\
& \sum m_{v_{f}} \cdot v_{x, f}+\sum m_{v_{f}} \cdot v_{b, f}+m_{X}(X)=\sum m_{v_{f}} \cdot v_{y, f}+\sum m_{v_{f}} \cdot v_{z, f}+\sum m_{v_{f}} \cdot v_{d_{i, f}}+\sum m_{v_{f}} \cdot v_{c, f}+m_{Y}(Y)+m_{Z}(Z)
\end{aligned}
$$

$m_{v_{f}}$ is the neutrino mass for different flavors, $m_{X}(X)$ is the mass of target nucleus. $m_{Y}(Y)$ and $m_{Z}(Z)$ are the masses of fission fragments. However, if the neutrino oscillation phenomena occurs, the invariant masses of the neutrinos might not get conserved, as different neutrino flavors have distinct invariant masses, with the first approximation-independent upper bound with the lightest mass ${ }^{13}$ to be $<0.086 \mathrm{eV}(95 \% \mathrm{CI})$ and sum of neutrino masses $\sum m_{v_{f}} \lesssim$ $0.26 \mathrm{eV}(95 \% \mathrm{CI})$. In addition, neutrino velocity can change upon scattering with target nucleus, and the velocity of target nucleus is negligibly changed to conserve the momentum of the reaction.

On the premise of Process 1, which is the dynamic combination of neutrino(s) scattering and capturing. The energy states $E_{X, i}$ of target nucleus are fluctuating, governed by neutrino number density, energy of neutrons, and microscopic cross sections. The degree of freedoms involved in the evaluation of these energy states are immensely huge and lacks technological advancements. To serve this purpose, the concepts of statistical mechanics are incorporated to evaluate probability distribution $P\left(E_{X, i}\right)$ of these energy state fluctuations of the target nucleus.

\section{Mathematical Formulation}

In this section, the probability distribution of energy states $P\left(E_{X, i}\right)$ of target nucleus $X$ by neutrino interactions is derived. Consider a system which comprises of two subsystems 1and 2, shown in Figure 1. The volume of system is fixed and has the total energy $E_{t o t}$ and total number of microstates $\Omega_{t o t}\left(E_{t o t}\right)$.

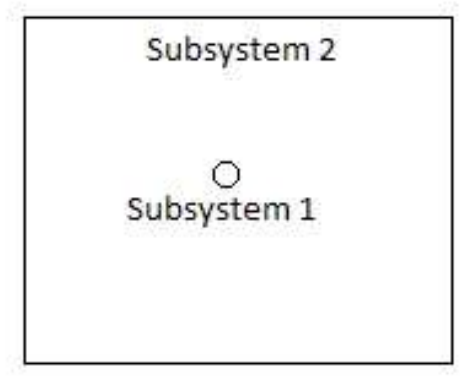

Figure 1: System with fixed volume with two subsystems 1 and 2.

\subsection{Subsystems}

Subsystem 1 comprises of a target nucleus at rest. The energy of subsystem 1 is $E_{X}$ and has the microstates $\Omega_{X}\left(E_{X}\right)$. Subsystem 2 represents a neutrino bath with energy $E_{v}$ and microstates $\Omega_{v}\left(E_{v}\right)$. The total energy of the system is the sum of two subsystems 1 and 
2 , given by equation 8 . The total microstates of the system is the product of microstates of subsystems 1 and 2, given by equation 9 .

$$
\begin{aligned}
& E_{t o t}=E_{X}+E_{v} \\
& \Omega_{t o t}\left(E_{t o t}\right)=\Omega_{X}\left(E_{X}\right) \cdot \Omega_{v}\left(E_{v}\right)
\end{aligned}
$$

\subsection{Assumptions}

Two subsystems 1 and 2 are in a state of equilibrium, where the probability of all accessible microstates is equal. Furthermore, the energy of subsystem 1 is exceedingly less than the total energy i.e. $E_{X}<<<E_{\text {tot }}$. The total number of neutrinos in subsystem 2 is considerably large and fixed. These are reasonable assumptions, as the target nucleus is at rest and the number of neutrinos is considerably large. In Supernova, the electron neutron flux is of the order $10^{42}$ neutrinos $/ \mathrm{cm}^{2}$, with average energy ${ }^{1}$ of $10 \mathrm{MeV}$. In addition, assume the energy states of neutrinos are continuum and $\frac{\partial \ln \Omega_{v}\left(E_{t o t}\right)}{\partial E_{t o t}}$ is well defined. Based on these assumptions, it is logical to assume that subsystem 2 governs the fluctuations of subsystem 1 and subsystem 1 has negligible effect on subsystem 1 .

\subsection{Probability Distribution $P\left(E_{X, i}\right)$}

Based on the assumptions, the probability $P\left(E_{X}\right)$ i.e. the number o microstates of total system such that energy of subsystem 1 is $E_{X}$ over the total number of microstates is given by equation 10 .

$P\left(E_{X}\right)=\frac{\Omega_{v}\left(E_{v}\right)}{\Omega_{\text {tot }}\left(E_{\text {tot }}\right)}$

Taking natural logarithm of equation 10 and using equation 8 , it can be written as

$\ln P\left(E_{X}\right)=\ln \Omega_{v}\left(E_{t o t}-E_{X}\right)-\ln \Omega_{t o t}\left(E_{t o t}\right)$

Since we have assumed $E_{X}<<<E_{\text {tot }}$, the first term of equation 11 is expanded using Taylor series at energy $E_{\text {tot }}$. This equation can be written as equation 12 .

$\ln P\left(E_{X}\right)=\ln \Omega_{v}\left(E_{t o t}\right)-\left.E_{X} \frac{\partial \ln \Omega_{v}\left(E^{\prime}\right)}{\partial E^{\prime}}\right|_{E^{\prime}=E_{t o t}}+H . T-\ln \Omega_{t o t}\left(E_{t o t}\right)$

Neglecting higher order terms $(H . T)$ and representing constant terms with $\mathrm{m}$ where $m=\ln \Omega_{v}\left(E_{t o t}\right)-\ln \Omega_{t o t}\left(E_{t o t}\right)$, equation 12 can be written as

$\ln P\left(E_{X}\right)=m-E_{X} \cdot \zeta$

where $\zeta=\left.\frac{\partial \ln \Omega_{v}\left(E^{\prime}\right)}{\partial E^{\prime}}\right|_{E^{\prime}=E_{t o t}}$ is Neutrino-Zeta defined in section 3.4

Taking natural logarithm of equation 13 , the probability $P\left(E_{X}\right)$ is written as

$P\left(E_{X}\right)=s \cdot e^{-E_{X} \cdot \zeta}$

$\mathrm{S}$ in equation 14 is given by $s=e^{m}=\frac{\Omega_{v}\left(E_{t o t}\right)}{\Omega_{t o t}\left(E_{t o t}\right)}$, which is the ratio of microstates of subsystem 2 with energy $E_{\text {tot }}$ and total number of microstates. 
The precise deterministic energy states and energy levels of subsystem 1, target nucleus $X$ in this case are governed by the dynamics of nucleus itself, which is a separate quantum mechanical study. However, in this article the energy states of subsystem 1 are reasonably assumed to be discrete i.e. $E_{X, i}$. Therefore, the normalized probability distribution $P\left(E_{X, i}\right)$ is given by equation 15 .

$P\left(E_{X, i}\right)=\frac{s \cdot e^{E_{x, i} \cdot \zeta}}{\left.\sum_{\text {states } j=1}^{\left(e^{-E_{X, j} \cdot \zeta}\right.}\right)}$

$\sum\left(e^{-E_{X, j} \cdot \zeta}\right)$ in equation 15 is the partition function, which normalizes the probability states $j=1$

distribution over all energy states of subsystem 1 . The partition function is defined by $Z=\sum_{\text {states } j=1}\left(e^{-E_{X, j} \cdot \zeta}\right)$, such that the probability distribution is given by equation 16 .

$P\left(E_{X, i}\right)=\frac{s \cdot e^{E_{x, i} \cdot \zeta}}{Z}$

\subsection{Neutrino-Zeta $\zeta$}

Neutrino-Zeta $\zeta$ is a quantity which governs the fluctuations in the energy states of subsystem 1 . Neutrino-zeta is only dependent on properties of subsystem 2 and has the units of inverse of energy $\left[\mathrm{eV}^{-1}\right]$. It is defined as

$\zeta=\frac{k}{\sum n v_{f} \cdot<e_{v}>\cdot \sigma_{v}}$

$\sum n v_{f}$ is the number of neutrinos per unit area i.e. neutron number density, in the neutrino bath. The number density is the combined number of all flavors of neutrinos. $\left\langle e_{v}\right\rangle$ is the average kinetic energy of a single neutrino, given in $\mathrm{eV}$ and $\sigma_{v}$ is the total microscopic cross-section of neutrinos given in units of Barns or $\mathrm{cm}^{-2}$. The total microscopic cross-section is the sum of scattering cross-section and capture crosssection. Furthermore, the capture and scattering cross-sections are different for each neutrino flavor. Therefore, the total cross-section is given by equation 18 .

$\sigma_{v}=\sum \sigma_{s, f}+\sum \sigma_{c, f}$

Where frepresents the neutrino flavor.

$k$, in the definition of neutrino-zeta is Naeem's Constant, which is dimensionless constant of proportionality that links entropy $S_{v}$ and microstates $\Omega_{v}$ of neutrino-bath, such that

$S_{v}=\frac{1}{k} \ln \Omega_{v}$

Therefore,

$\left.\frac{\partial S_{v}}{\partial\left(E^{\prime}\right)}\right|_{E^{\prime}=E_{t o t}} \approx \frac{1}{\sum n v_{f} \cdot<e_{v}>\cdot \sigma_{v}}$

The numerical value of $k$ is estimated in section 4 . The significance of zeta lies in the fact that it governs the probability distribution of energy states of subsystem 1, in this case a target nucleus. 


\subsection{Fission Barrier Energy State $E_{X, f}$}

Fission Barrier energy state is the minimum energy state at which the target nucleus is irretrievably committed for fission process. Based on the mathematical formulation, the probability of fission barrier energy state $P\left(E_{X, f}\right)$ is given by

$P\left(E_{X, f}\right)=\frac{s \cdot e^{-E_{x, f} \cdot \zeta}}{\sum_{\text {states } j=1}\left(e^{-E_{X, j} \cdot \zeta}\right)}$

At energy states higher than fission barrier energy states i.e. $E_{X, i}>E_{X, f}$, fission process is also favorable. The higher the energy state, more the target nucleus is excited. Higher excitation energy tends towards symmetric fission fragments. The probability distribution function of energy states $P\left(E_{X, i}\right)$ is a decreasing exponential function of neutrino-zeta $\zeta$. Therefore, for one neutrino-zeta, the probabilities of energy states higher than fission barrier energy state are exponentially decreasing, as shown in figure 2. For different neutrino-zetas, the probability distribution curves will be different, hence the probabilities of fission fragments once the target nucleus undergoes fission.

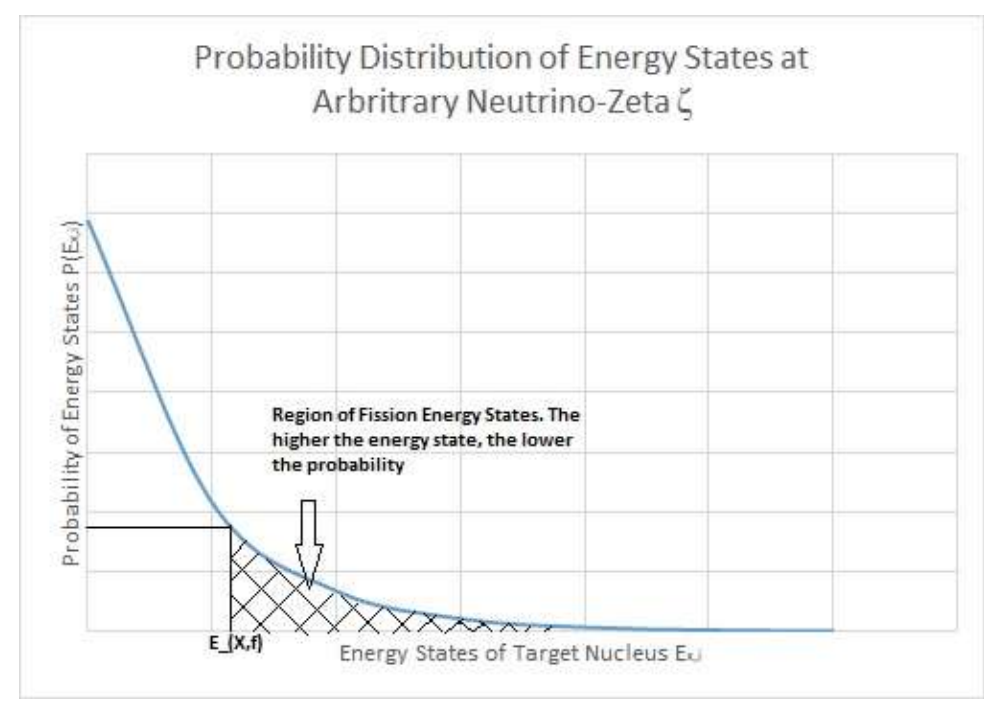

Figure 2: Probability Distribution of Energy States

\section{Estimation of Neutrino-Zeta $\zeta$}

In this section, the neutrino-zeta is estimated in supernovas which are r-process sites, where neutrino-captured heavy nuclei fission process is a common occurrence. The neutrino number density is extremely high crudely of the order $5 \times 10^{42} \mathrm{~cm}^{2}$, in these sites. Neutrino-induced charged current total cross-section ${ }^{1}$ on U-238 is approximately $\sigma_{v}=500 \cdot 10^{-42} \mathrm{~cm}^{-2}$, which assumes Fermi-Dirac spectrum with temperature $T_{v}=4 \mathrm{MeV}$ and zero chemical potential for electron neutrinos.

\subsection{Assumptions}

For the estimations of neutrino-zeta, the charged-current electron neutrinos with average energy $<e_{v}>\sim 10 \mathrm{MeV}$, interacts with heavy nuclei with $A \sim 230-270$. 
The neutral-current charged reactions of muon and tau neutrinos can also occur in the core bounce supernova explosions with neutrino average energies of $\left\langle e_{v}\right\rangle \sim 20-$ $25 \mathrm{MeV}$. The fission probability ${ }^{14}$ as a function of Fission Barrier, neutron separation energy and Temperature is approximately given by equation 22 .

$P_{f}=\frac{1}{1+4\left(m_{n} / \hbar^{2}\right) R^{2} T e \frac{B^{-S_{n}}}{T}}$

$m_{n}$ is the nucleon mass, $B_{f}$ is the Fission Barrier, $S_{n}$ is the Neutron Separation energy, $T$ is the Temperature and $R=1.2 \cdot A^{\frac{1}{3}}$. Equation 22 assumes, the decaying nucleus is excited at energy higher than Fission Barrier and Neutron Separation energies. Supernova neutrinos captured on heavy target nuclei, where excitation energies are about $E \sim 25 \mathrm{MeV}$, which corresponds to Temperature $T \sim 1 \mathrm{MeV}$ for heavy nuclei with $A \sim 230-270$. Based on these values, the fission probability given by equation 22 is approximately $P_{f} \approx \frac{1}{6}$ when $B_{f}=S_{n}$. Assuming, the fission probability of equation 22 is equal to the probability of fission barrier energy state of target heavy nuclei. i.e. $P\left(E_{X, f}\right) \approx P_{f}$. Using equation 21 ,

$P\left(E_{X, f}\right)=\frac{s \cdot e^{-E_{X, f} \cdot \zeta}}{\sum_{\text {states } j=1}\left(e^{-E_{X, j^{\prime}} \cdot \zeta}\right)} \approx P_{f}$

The energy state of the target nucleus depends on the dynamics of the nucleus and are assumed to be discrete. To estimate partition function, the energy states are assumed to be $n$, where $n=7$. The energy states are assumed to be $[0,5,10,15,20,25,30] \mathrm{MeV}$. The partition function is the function of neutrino-zeta $\zeta$ and not the energy states of the nucleus.

\subsection{Neutrino-Zeta Calculation}

In order to calculate neutrino-zeta, the fission barrier energy state can be written using equation 23 as

$s \cdot e^{-E_{X, f} \cdot \zeta} \approx P_{f}\left[\sum_{\text {states } j=1}^{-E X, j} \cdot \zeta\right]$

$s$ is the ratio of $\frac{\Omega_{v}\left(E_{t o t}\right)}{\Omega_{t o t}\left(E_{t o t}\right)}$ can be reasonably assumed to be crudely 1 i.e. $s \approx 1$.

Applying Maclaurin series of exponential functions, equation 24 can be written as

$$
\begin{aligned}
1+\left(-E_{X, f} \cdot \zeta\right) & +\frac{\left(-E_{X, f} \cdot \zeta\right)^{2}}{2}+H \cdot T \\
& \approx P_{f}\left[1+\left(-E_{X, 1} \cdot \zeta\right)+\frac{\left(-E_{X, 1} \cdot \zeta\right)^{2}}{2}+H \cdot T+1+\left(-E_{X, 2} \cdot \zeta\right)\right. \\
& \left.+\frac{\left(-E_{X, 2} \cdot \zeta\right)^{2}}{2}+H \cdot T+1+\left(-E_{X, n} \cdot \zeta\right)+\frac{\left(-E_{X, n} \cdot \zeta\right)^{2}}{2}+H \cdot T\right]
\end{aligned}
$$

Neglecting higher order terms in equation 25, the second order quadratic equation for neutrino-zeta can be written as 


$$
\zeta^{2}\left[\frac{\left(-E_{X, f}\right)^{2}}{2}-P_{f}\left\{\frac{\begin{array}{c}
\sum\left(-E_{X, j}\right)^{2} \\
2
\end{array}}{2}\right\}+\zeta\left[P_{f}\left\{\begin{array}{c}
n \\
\left.\sum E_{X, j}\right\} \\
j
\end{array}\right\}-E_{X, f}\right]=P_{f}[n]-1\right.
$$

Where $n$ the number of energy are states and $E_{X, j}$ are the energy states where $j=$ $1,2, \ldots n$. The neutrino-zeta is therefore given by equation 27

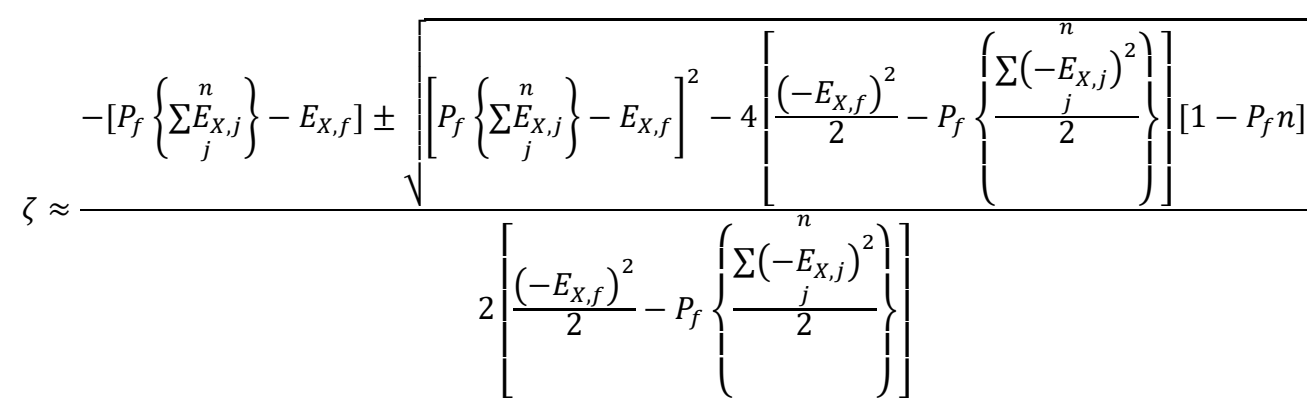

The fission Barrier Energy State $E_{X, f}$ is assumed to be $6 \mathrm{MeV}$, which is approximately the fission Barrier of Uranium-238. Based on the assumed values of energy states and number of energy states. The neutrino-zeta is approximately $\zeta \approx 0.0212$, which is based on the second order approximation of Maclaurin series of exponential function. The first order approximation of Maclaurin series of exponential function came to be 0.0146 . The other value of neutrino-zeta using equation 27 is approximately 0.045 , which was neglected for preceding calculations, because it diverges more from first order approximation. The partition function based on the assumed energy states and number is 5.208427. The Probability Distribution of energy states of target nucleus with $6 \mathrm{MeV}$ fission barrier is shown in Figure 3. The probability of fission Barrier energy state using equation 23 is $P\left(E_{X, f}\right)=0.169064014$, which is approximately equal to $P_{f}$. Using equation 17, the approximate value of Naeem's Constant $k$ is crudely equal to $k \approx \zeta \cdot\left[n v_{e} \cdot\left\langle e_{v}>\cdot \sigma_{v}\right] \approx 530\right.$ 


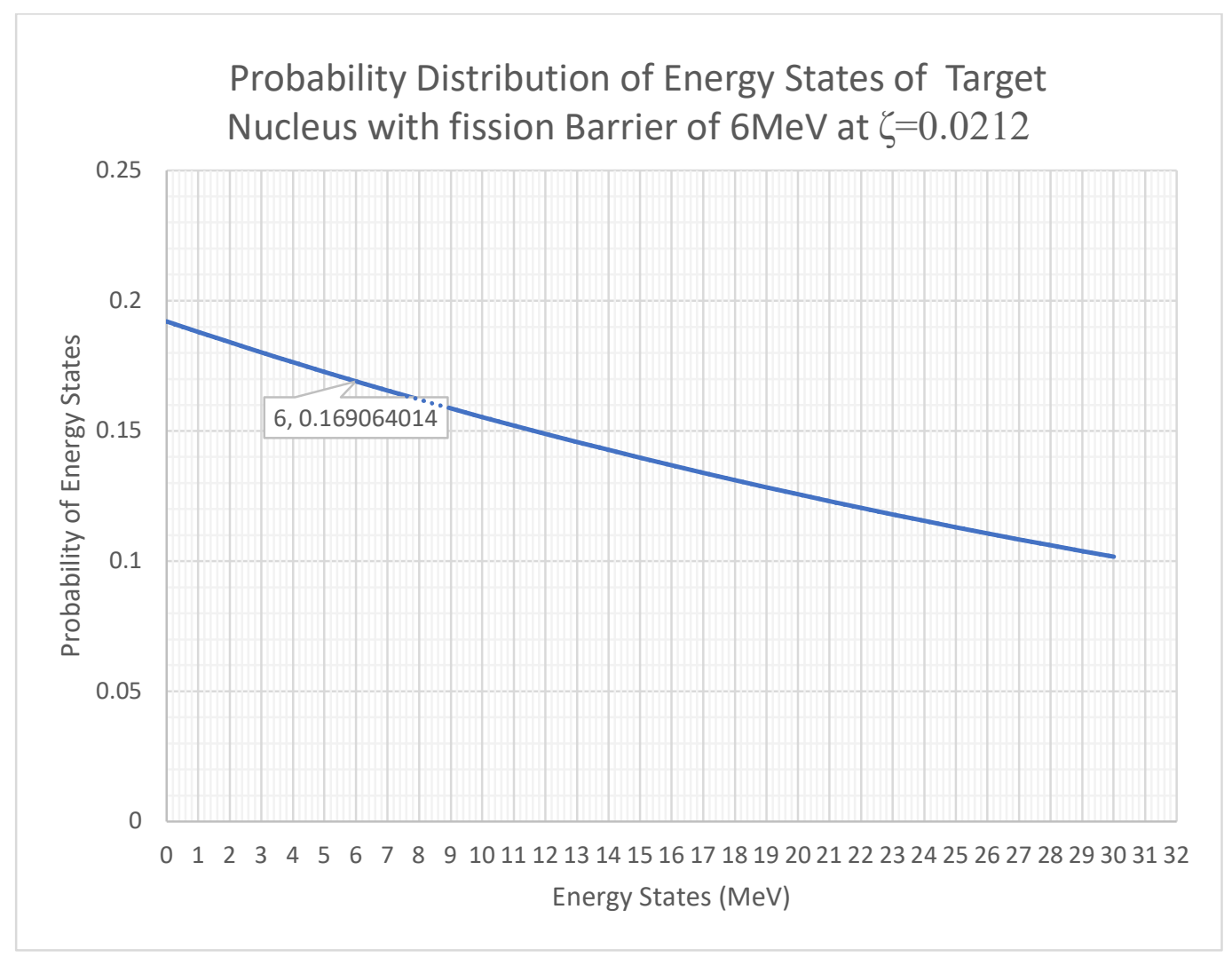

Figure 3. Probability Distribution of Energy States at $\zeta=0.0212$

\subsection{Effect of Neutrino-Zeta on $P\left(E_{X, i}\right)$}

In this section, the effect of neutrino-zeta on the probability distribution of energy states $P\left(E_{X, i}\right)$ of target nucleus is presented. The probability distribution of energy states at different neutrino-zetas is shown in Figure 4 and Figure 5 


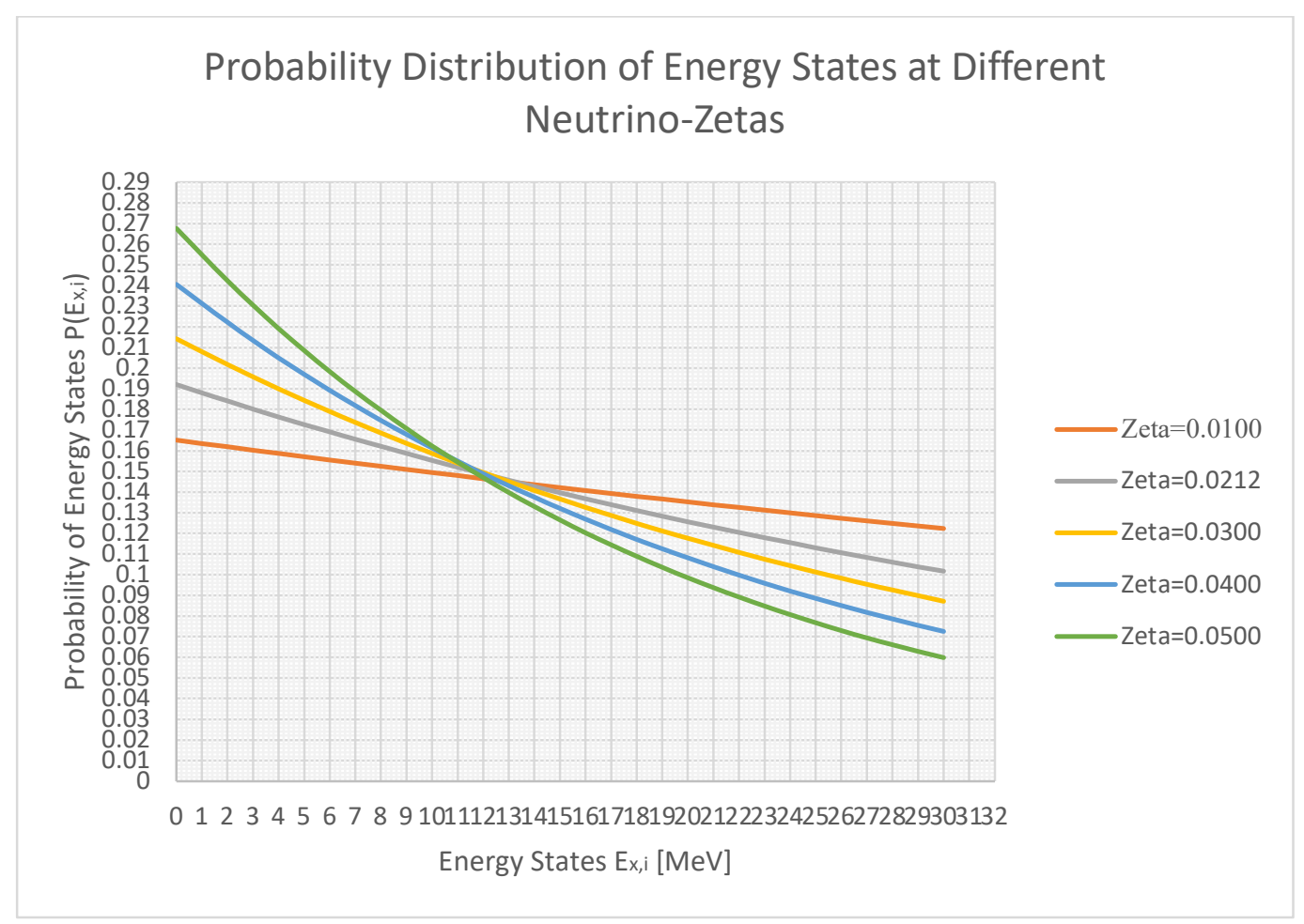

Figure 4. Probability Distribution at Different Neutrino-Zeta Values

The estimated neutrino-zeta at Supernova with assumed conditions is $0.0212 \mathrm{MeV}^{-1}$. Figure 4 indicates that probability distribution curves at different neutrino-zeta values intersects at a certain energy state. For example, the probability distribution curves in Figure 4 at $\zeta=0.0212$ and $\zeta=0.0100$ intersects at energy state of about $13.6 \mathrm{MeV}$, with the $\mathrm{P}(13.6)$ is about 0.145 . Likewise, the distribution curves in Figure 5 at $\zeta=$ 0.0212 and $\zeta=0.5000$ intersects at about $3.3 \mathrm{MeV}$ with $\mathrm{P}(3.3)$ about 0.18 . If these intersection activation energy state corresponds to a certain reaction, the probability of occurrence of that reaction is same at both Neutrino-Zetas. 


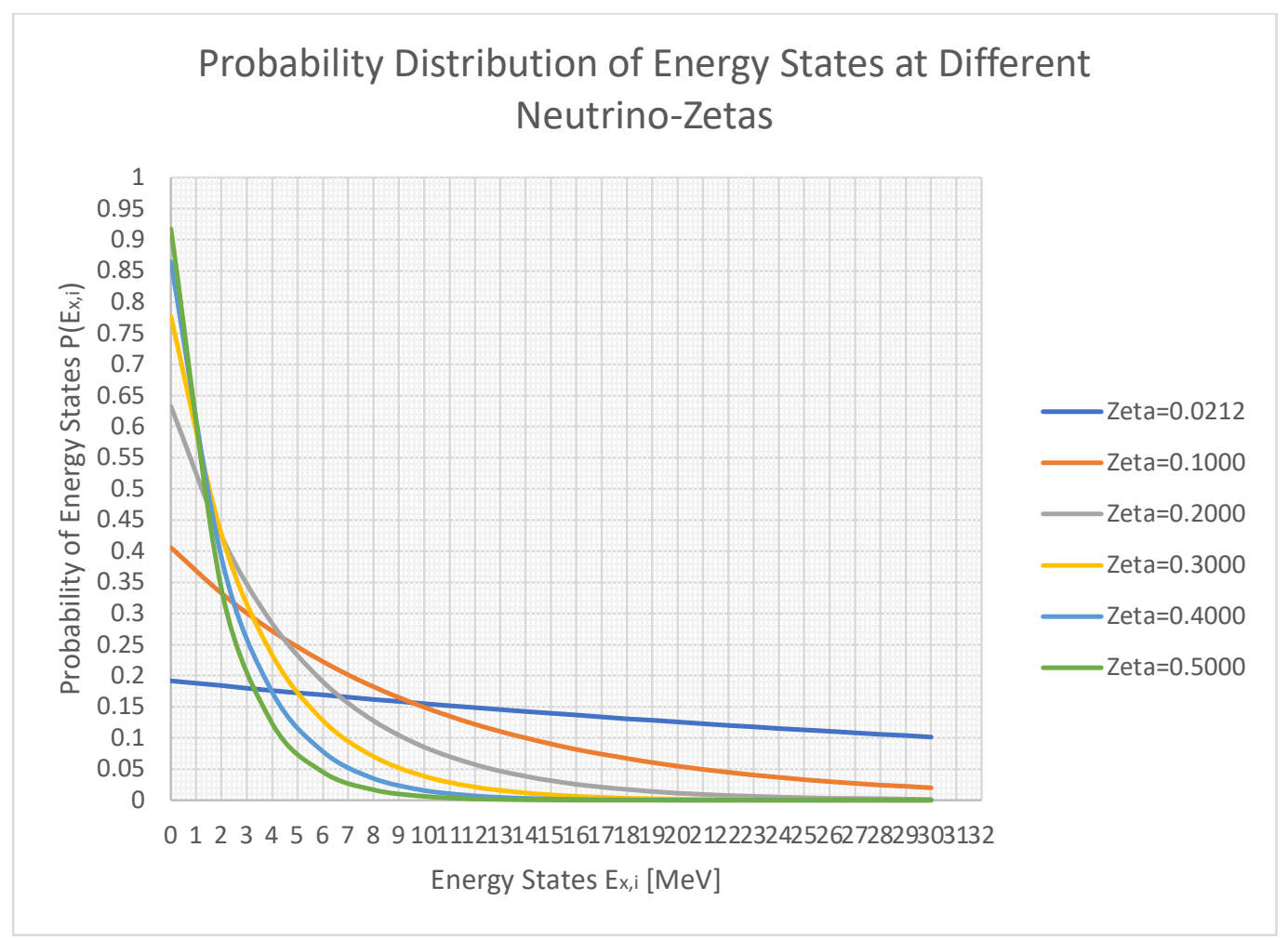

Figure 5: Probability Distribution at Different Neutrino-Zeta Values

\subsection{Points of Intersection}

The importance of point of intersection lies in the fact that at intersection point of two probability distribution curves, the probability of energy state is equal. It signifies that in different neutrino environments, the probability of an arbitrary reaction, which occurs at activation energy equals to the intersection energy state is same i.e. $P\left(E_{R}\right)=$ $P\left(E_{\text {int }}\right)$ where $R$ is an arbitrary reaction occurring at activation energy $E_{R}$ and $E_{\text {int }}$ is the intersection energy state of two neutrino-zetas. Furthermore, the relationship between energy states and neutrino-zetas depends on whether the energy state in question is more or less than the intersection energy state $E_{\text {int }}$. If energy state in question i.e. $E_{x}, \quad$ is greater than $E_{\text {int }}$, then the probability of energy state $\mathrm{P}\left(E_{x}\right)$ increases with increasing neutrino-zeta. Similarly, if $E_{x}<E_{\text {int }}$ then $\mathrm{P}\left(E_{x}\right)$ increases with increasing neutrino-zeta. The probabilities of target nucleus with fission barrier $E_{x}=$ $E_{X, f}=6 \mathrm{MeV}$ at various zeta is shown in Figure 6. 


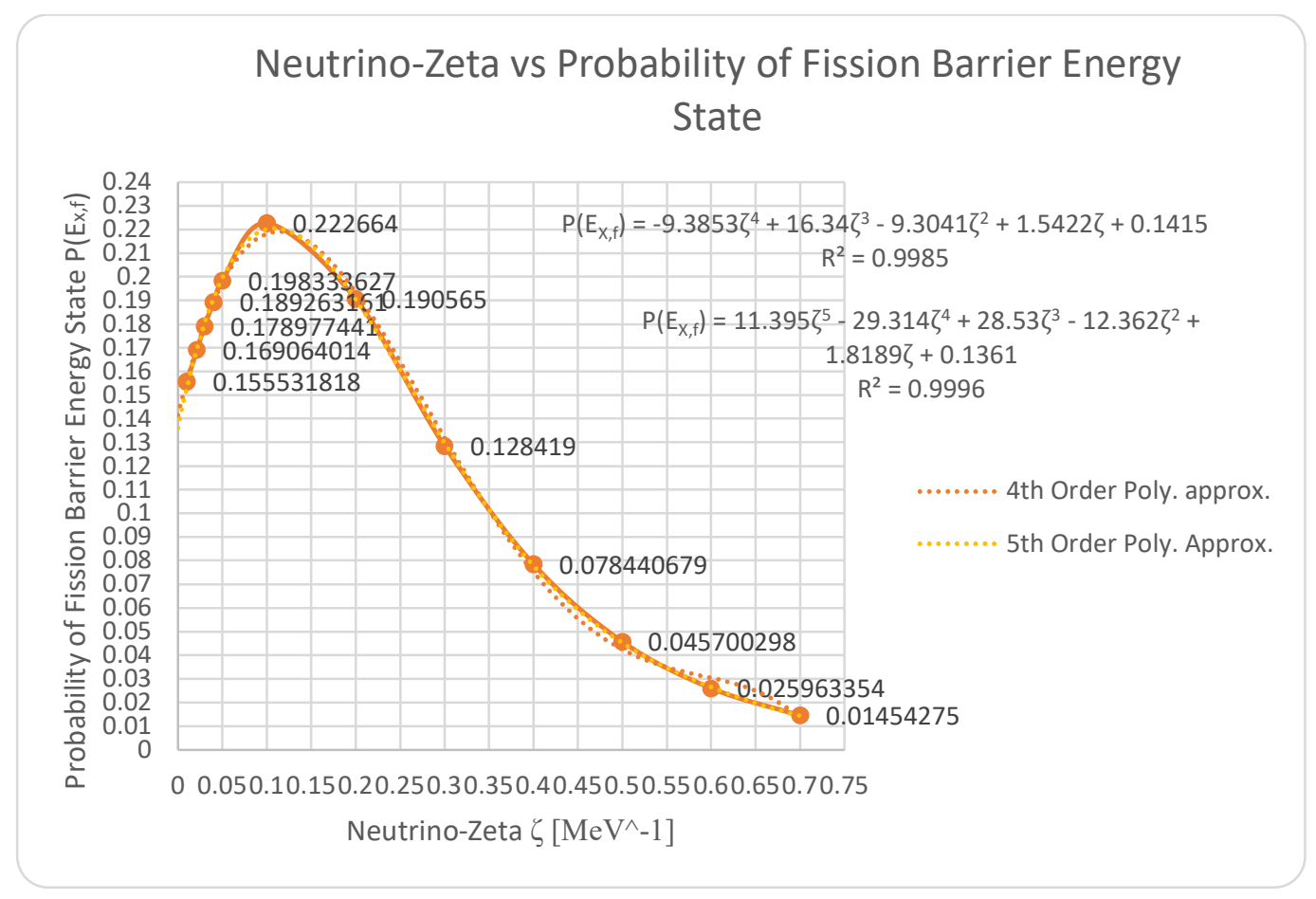

Figure 6: Probability of Fission Barrier Energy State at various Neutrino-Zetas. $\mathrm{P}\left(E_{X, f}\right)=6 \mathrm{MeV}$

The probability of fission barrier energy state $\mathrm{P}\left(E_{X, f}\right)=6 \mathrm{MeV}$ increases with neutrinozeta initially, and reaches the maximum at around 0.1 neutrino-zeta, with the probability of about 0.222664 . The probability values start to fall after the maxima at 0.1 neutrinozeta. The fourth and fifth order approximations are shown in Figure 6, with the Rsquared values of 0.9985 and 0.9996 respectively. Similarly, the probabilities of various energy states at different neutrino-zeta values are shown in Figure 7 and Table 1 in Appendix A. On the premise that these energy states correspond to different nuclear reactions, the probabilities of certain reactions are a function of neutrino-zeta. 


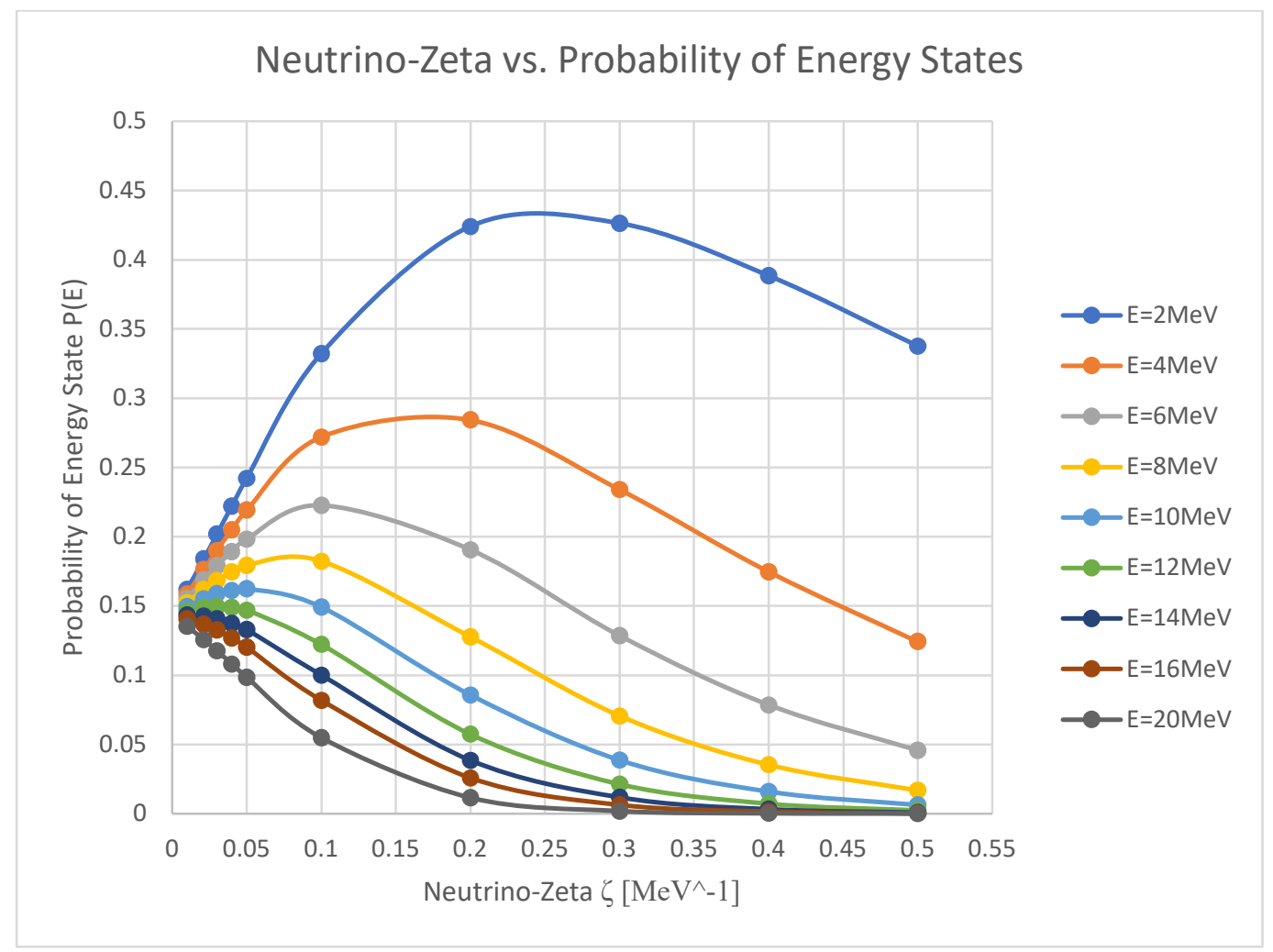

Figure 7. Probabilities of Target Nucleus Energy States at Various Neutrino-Zetas

\section{Neutron induced Fission Reaction in Neutrino-Zeta}

This section presents nuclear reaction for induced fission of an arbitrary target nucleus at rest with free neutron with negligible kinetic energy in neutrino-zeta. The nuclear reaction is given by equation 28 .

$\sum a_{f} v_{f}+X+n \stackrel{1}{\rightarrow}[X]^{*}+\sum b_{f} v_{f} \stackrel{2}{\rightarrow}[Y]^{*}+[Z]^{*}+\sum_{i}^{m}\left[n_{i}\right]^{*}+\sum c_{f} v_{f}$

$[X]^{*}$ in this reaction is given by $[X]^{*}=X+n+\sum x_{f} v_{f}$, the remaining quantities are defined in section 1. The number of neutrinos is conserved in this reaction. Furthermore, mass, momentum and energy in this reaction is also conserved.

\subsection{Q-Value of the reaction}

Q-value represents difference between the initial and final mass of the reaction. The initial and final masses of the reaction are given by equation 29 and 30 respectively.

$$
\begin{gathered}
M_{I} c^{2}=\left[\left(Z m_{p}+N m_{n}\right)+\left(\sum m_{v_{f}} \cdot v_{x, f}\right)\right] c^{2}-(B . E)_{X^{*}} \\
M_{F} c^{2}=\left[\left(\left(M_{Y}\right) c^{2}-(B \cdot E)_{Y}\right)+\left(\left(M_{Z}\right) c^{2}-(B . E)_{Z}\right)+\left(\left(M_{1}\right) c^{2}-(B . E)_{n}\right)+\right. \\
\left.\left(\left(\sum m_{v_{f}} \cdot v_{y, f}+\sum m_{v_{f}} \cdot v_{z, f}+\sum m_{v_{f}} \cdot v_{d_{i, f}}\right) c^{2}-(B . E)_{v}\right)\right]
\end{gathered}
$$


B.E is the respective binding energy, $M_{Y}=Z_{Y} m_{p}+N_{Y} m_{n}, M_{Z}=Z_{Z} m_{p}+N_{Z} m_{n}$ and $M_{1}=m_{n}[i], i$ are the number of neutrons released. The Q-Value of the reaction is given by equation 31 .

$Q=\left(M_{I}-M_{F}\right) c^{2}$

Since, the nucleon number is conserved in process 2 , and assuming no further neutrinos are captured during the fission process such that

$\sum m_{v_{f}} \cdot v_{x, f}=\sum m_{v_{f}} \cdot v_{y, f}+\sum m_{v_{f}} \cdot v_{z, f}+\sum m_{v_{f}} \cdot v_{d_{i, f}}$

The Q-value only depends on the Binding energies. The Binding energy for neutron is 0 , and binding energy of neutrino(s) is assumed to be 0 . i.e. $(B . E)_{n}=0$ and $(B . E)_{v}=0$. The Qvalue is therefore given by $Q=(B . E)_{Y}+(B . E)_{Z}-(B . E)_{X^{*}}$, which is generally the case in past literature on fission reaction. Neutrinos does not affect the Q-value of the reaction, regardless of the number of neutrinos captured and their energies for any specific reaction.

\subsection{Excitation of Target Nucleus}

The Q-Value of Process 2 is unaffected by the number of neutrinos captured, however, Process 1 is affected by number of neutrinos captured, their energies and reaction crosssections. More the number of neutrinos captured, more than excitation energy of the target nucleus $\mathrm{X}$. It is common knowledge that excitation energy of the target nucleus affects the fission fragments. At higher excitation energies, fission fragments tend to be symmetric and the fission fragment probability distribution is changed. Based on this premise, the number of neutrinos captured in process 1 affects the fission fragment probability distribution. Using equation 16, the probability distribution of energy states by neutrino(s) interaction is estimated.

\section{Assumptions}

To estimate the probability distribution, it has been assumed that the total activation energy for the target nucleus is the sum of activation by free neutron with negligible kinetic energy capture and the activation by neutrino(s) interaction, given by equation 33.

$E^{*}=E_{n}^{*}+E_{v}^{*}$

$E_{n}^{*}$ is the activation energy by neutron capture and is assumed to be equal to fission barrier of target nucleus. $E_{n}^{*}=E_{X, f}$. This assumption implies fission reaction is inevitable regardless of the neutrino(s) interaction. $E_{v}^{*}$ is the activation energy by neutrino(s) interaction. Distinct energy states of Target nucleus depends on the mechanics of the nucleus itself, so $E_{v}^{*}$ values of Target Nucleus are assumed, given in Table 2 Appendix A. The probability distribution of neutrino(s) interaction activation energy states $P\left(E_{v}^{*}\right)$ at different neutrino-zeta values are shown in Figure 8. 


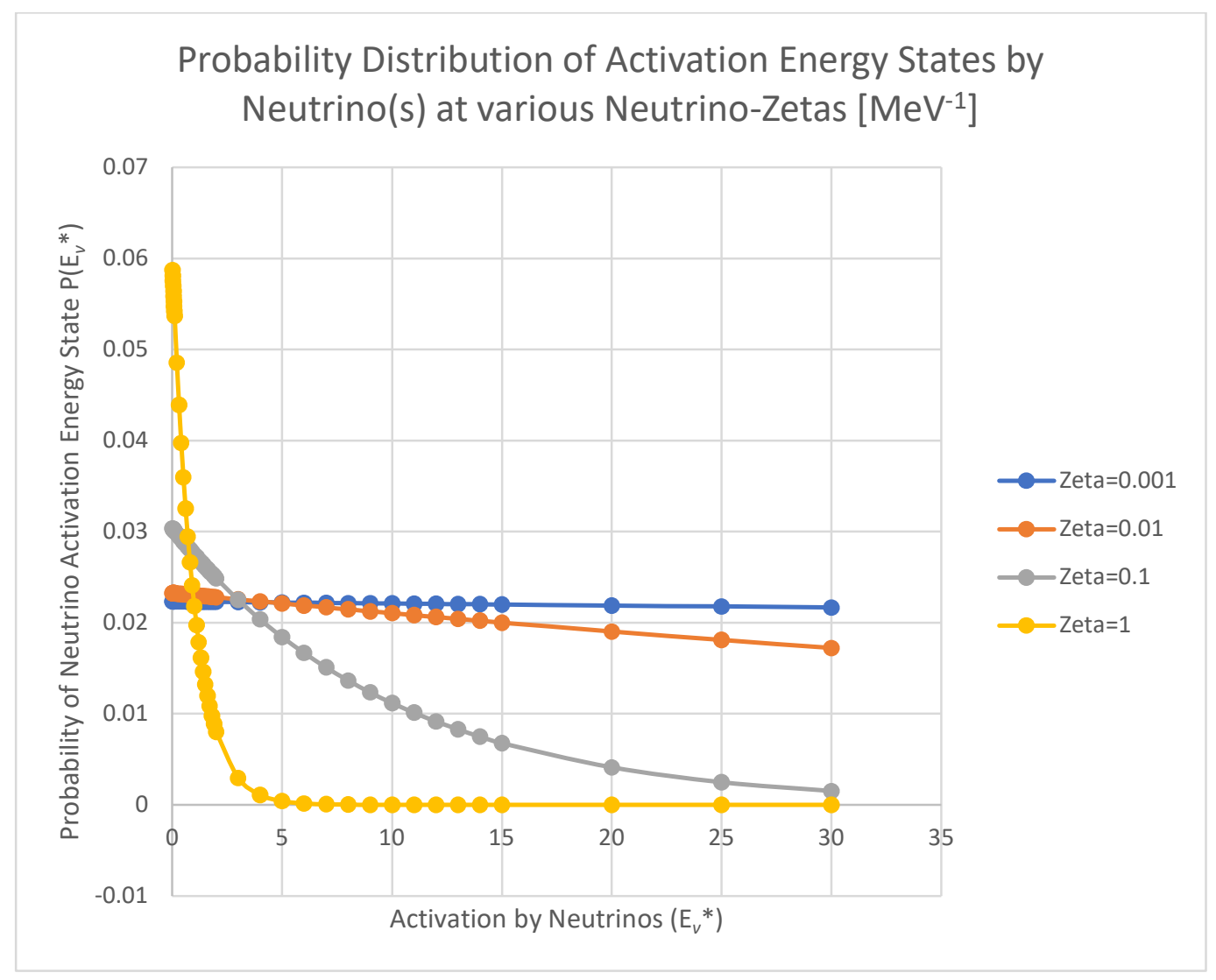

Figure 8. Probability Distribution of Neutrino-interaction activation energy states

Figure 8 indicates that Target nucleus with the Fission Barrier energy state by neutron will have additional probability of excitation because of neutrino(s) interactions. Assuming these distinct activation energy states corresponds to distinct fission fragments of target nucleus, lower neutrino-zeta values have higher probabilities of symmetric fission Channel. At $\zeta=0.001$, the probabilities of all excitation energy states are approximately equal. Target nucleus undergoing neutron induced fission in that neutrino environment conditions, the probability of symmetric and asymmetric fissions is approximately equal, and as neutrino-zeta increases, the probability of asymmetric channels is higher.

\section{Conclusion}

It has been shown that neutrino(s) interactions affect the excitation energy states of heavy nuclei, which are statistically quantifiable by neutrino-zeta $\zeta$, a quantity of neutrino environments. This quantity can be vastly used in the supernova sites, which neutrino fluxes are very high. Future prospect would be to quantify the excitation energy states of heavy nuclei and their interaction cross-sections with neutrinos, which can be done experimentally or theoretically. Furthermore, it has been shown that certain decay reaction probabilities are equal at different neutrino-zetas. In addition, it has been shown, given exact excited energy states, the fission fragments probabilities for neutrino fission and neutron induced fission in neutrino environments can be different. 
It is hypothesized that at lower neutrino-zetas, the probabilities of symmetric fission fragments are higher, which can be experimentally tested using different neutrino-zeta values.

\section{Appendix A}

\begin{tabular}{|c|c|c|c|c|c|c|c|c|c|c|}
\hline $\begin{array}{l}\text { (E) } \\
\mathrm{MeV}\end{array}$ & $\zeta=0.01$ & $\begin{array}{l}\zeta= \\
0.0212\end{array}$ & $\zeta=0.03$ & $\zeta=0.04$ & $\zeta=0.05$ & $\zeta=0.1$ & $\zeta=0.2$ & $\zeta=0.3$ & $\zeta=0.4$ & $\zeta=0.5$ \\
\hline $\mathrm{P}(2)$ & $\begin{array}{l}0.16187 \\
9\end{array}$ & $\begin{array}{l}0.18402 \\
6\end{array}$ & $\begin{array}{l}0.20179 \\
7\end{array}$ & $\begin{array}{l}0.22210 \\
2\end{array}$ & $\begin{array}{l}0.24224 \\
5\end{array}$ & $\begin{array}{l}0.33217 \\
6\end{array}$ & 0.42411 & $\begin{array}{l}0.42636 \\
7\end{array}$ & $\begin{array}{l}0.38851 \\
9\end{array}$ & $\begin{array}{l}0.33768 \\
2\end{array}$ \\
\hline $\mathrm{P}(4)$ & $\begin{array}{l}0.15867 \\
4 \\
\end{array}$ & $\begin{array}{l}0.17638 \\
6 \\
\end{array}$ & $\begin{array}{l}0.19004 \\
5 \\
\end{array}$ & $\begin{array}{l}0.20502 \\
6 \\
\end{array}$ & $\begin{array}{l}0.21919 \\
3 \\
\end{array}$ & $\begin{array}{l}0.27196 \\
3 \\
\end{array}$ & $\begin{array}{l}0.28428 \\
9 \\
\end{array}$ & $\begin{array}{l}0.23399 \\
5 \\
\end{array}$ & $\begin{array}{l}0.17457 \\
3\end{array}$ & $\begin{array}{l}0.12422 \\
6\end{array}$ \\
\hline $\mathrm{P}(6)$ & $\begin{array}{l}0.15553 \\
2 \\
\end{array}$ & $\begin{array}{l}0.16906 \\
4 \\
\end{array}$ & $\begin{array}{l}0.17897 \\
7 \\
\end{array}$ & $\begin{array}{l}0.18926 \\
3 \\
\end{array}$ & $\begin{array}{l}0.19833 \\
4 \\
\end{array}$ & $\begin{array}{l}0.22266 \\
4 \\
\end{array}$ & $\begin{array}{l}0.19056 \\
5 \\
\end{array}$ & $\begin{array}{l}0.12841 \\
9\end{array}$ & $\begin{array}{l}0.07844 \\
1 \\
\end{array}$ & 0.0457 \\
\hline $\mathrm{P}(8)$ & $\begin{array}{l}0.15245 \\
2 \\
\end{array}$ & $\begin{array}{l}0.16204 \\
6 \\
\end{array}$ & $\begin{array}{l}0.16855 \\
5 \\
\end{array}$ & $\begin{array}{l}0.17471 \\
2\end{array}$ & 0.17946 & $\begin{array}{l}0.18230 \\
2 \\
\end{array}$ & $\begin{array}{l}0.12773 \\
9 \\
\end{array}$ & $\begin{array}{l}0.07047 \\
8 \\
\end{array}$ & $\begin{array}{l}0.03524 \\
6 \\
\end{array}$ & $\begin{array}{l}0.01681 \\
2 \\
\end{array}$ \\
\hline $\mathrm{P}(10)$ & $\begin{array}{l}0.14943 \\
3\end{array}$ & $\begin{array}{l}0.15531 \\
8\end{array}$ & $\begin{array}{l}0.15873 \\
9\end{array}$ & $\begin{array}{l}0.16127 \\
9\end{array}$ & $\begin{array}{l}0.16238 \\
2\end{array}$ & $\begin{array}{l}0.14925 \\
6\end{array}$ & $\begin{array}{l}0.08562 \\
6\end{array}$ & $\begin{array}{l}0.03867 \\
9\end{array}$ & $\begin{array}{l}0.01583 \\
7\end{array}$ & $\begin{array}{l}0.00618 \\
5\end{array}$ \\
\hline $\mathrm{P}(12)$ & $\begin{array}{l}0.14647 \\
4\end{array}$ & $\begin{array}{l}0.14887 \\
1\end{array}$ & $\begin{array}{l}0.14949 \\
5 \\
\end{array}$ & 0.14888 & $\begin{array}{l}0.14692 \\
9\end{array}$ & $\begin{array}{l}0.12220 \\
1\end{array}$ & $\begin{array}{l}0.05739 \\
7\end{array}$ & $\begin{array}{l}0.02122 \\
8 \\
\end{array}$ & $\begin{array}{l}0.00711 \\
6\end{array}$ & $\begin{array}{l}0.00227 \\
5 \\
\end{array}$ \\
\hline $\mathrm{P}(14)$ & $\begin{array}{l}0.14357 \\
4\end{array}$ & 0.14269 & $\begin{array}{l}0.14078 \\
9\end{array}$ & $\begin{array}{l}0.13743 \\
3\end{array}$ & $\begin{array}{l}0.13294 \\
7\end{array}$ & 0.10005 & $\begin{array}{l}0.03847 \\
4\end{array}$ & 0.01165 & $\begin{array}{l}0.00319 \\
7\end{array}$ & $\begin{array}{l}0.00083 \\
7\end{array}$ \\
\hline $\mathrm{P}(16)$ & $\begin{array}{l}0.14073 \\
1\end{array}$ & $\begin{array}{l}0.13676 \\
7\end{array}$ & 0.13259 & $\begin{array}{l}0.12686 \\
7\end{array}$ & $\begin{array}{l}0.12029 \\
5\end{array}$ & $\begin{array}{l}0.08191 \\
4\end{array}$ & 0.02579 & $\begin{array}{l}0.00639 \\
4\end{array}$ & $\begin{array}{l}0.00143 \\
7\end{array}$ & $\begin{array}{l}0.00030 \\
8 \\
\end{array}$ \\
\hline $\mathrm{P}(20)$ & $\begin{array}{l}0.13521 \\
3\end{array}$ & $\begin{array}{l}0.12564 \\
7\end{array}$ & $\begin{array}{l}0.11759 \\
7\end{array}$ & $\begin{array}{l}0.10810 \\
9\end{array}$ & 0.09849 & $\begin{array}{l}0.05490 \\
8\end{array}$ & $\begin{array}{l}0.01158 \\
8\end{array}$ & $\begin{array}{l}0.00192 \\
6\end{array}$ & 0.00029 & $\begin{array}{l}4.17 \mathrm{E}- \\
05\end{array}$ \\
\hline
\end{tabular}

Table 1: Probabilities of Target Nucleus Energy States at Various Zetas.

\begin{tabular}{|l|l|l|l|l|}
\hline$\left(E_{v}^{*}\right) \mathrm{MeV}$ & $\zeta=0.001$ & $\zeta=0.01$ & $\zeta=0.1$ & $\zeta=1$ \\
\hline $\mathrm{P}(0.01)$ & 0.022327 & 0.023246 & 0.030345 & 0.058707 \\
\hline $\mathrm{P}(0.02)$ & 0.022327 & 0.023243 & 0.030314 & 0.058123 \\
\hline $\mathrm{P}(0.03)$ & 0.022327 & 0.023241 & 0.030284 & 0.057545 \\
\hline $\mathrm{P}(0.04)$ & 0.022326 & 0.023239 & 0.030254 & 0.056972 \\
\hline $\mathrm{P}(0.05)$ & 0.022326 & 0.023236 & 0.030223 & 0.056405 \\
\hline $\mathrm{P}(0.06)$ & 0.022326 & 0.023234 & 0.030193 & 0.055844 \\
\hline $\mathrm{P}(0.07)$ & 0.022326 & 0.023232 & 0.030163 & 0.055288 \\
\hline $\mathrm{P}(0.08)$ & 0.022326 & 0.023229 & 0.030133 & 0.054738 \\
\hline $\mathrm{P}(0.09)$ & 0.022325 & 0.023227 & 0.030103 & 0.054194 \\
\hline $\mathrm{P}(0.1)$ & 0.022325 & 0.023225 & 0.030073 & 0.053654 \\
\hline $\mathrm{P}(0.2)$ & 0.022323 & 0.023201 & 0.029773 & 0.048548 \\
\hline $\mathrm{P}(0.3)$ & 0.022321 & 0.023178 & 0.029477 & 0.043928 \\
\hline $\mathrm{P}(0.4)$ & 0.022318 & 0.023155 & 0.029184 & 0.039748 \\
\hline $\mathrm{P}(0.5)$ & 0.022316 & 0.023132 & 0.028893 & 0.035966 \\
\hline $\mathrm{P}(0.6)$ & 0.022314 & 0.023109 & 0.028606 & 0.032543 \\
\hline $\mathrm{P}(0.7)$ & 0.022312 & 0.023086 & 0.028321 & 0.029446 \\
\hline $\mathrm{P}(0.8)$ & 0.022309 & 0.023063 & 0.02804 & 0.026644 \\
\hline $\mathrm{P}(0.9)$ & 0.022307 & 0.02304 & 0.027761 & 0.024108 \\
\hline $\mathrm{P}(1)$ & 0.022305 & 0.023017 & 0.027484 & 0.021814 \\
\hline $\mathrm{P}(1.1)$ & 0.022303 & 0.022994 & 0.027211 & 0.019738 \\
\hline
\end{tabular}




\begin{tabular}{|l|l|l|l|l|}
\hline $\mathrm{P}(1.2)$ & 0.022301 & 0.022971 & 0.02694 & 0.01786 \\
\hline $\mathrm{P}(1.3)$ & 0.022298 & 0.022948 & 0.026672 & 0.01616 \\
\hline $\mathrm{P}(1.4)$ & 0.022296 & 0.022925 & 0.026407 & 0.014623 \\
\hline $\mathrm{P}(1.5)$ & 0.022294 & 0.022902 & 0.026144 & 0.013231 \\
\hline $\mathrm{P}(1.6)$ & 0.022292 & 0.022879 & 0.025884 & 0.011972 \\
\hline $\mathrm{P}(1.7)$ & 0.022289 & 0.022856 & 0.025626 & 0.010833 \\
\hline $\mathrm{P}(1.8)$ & 0.022287 & 0.022833 & 0.025371 & 0.009802 \\
\hline $\mathrm{P}(1.9)$ & 0.022285 & 0.02281 & 0.025119 & 0.008869 \\
\hline $\mathrm{P}(2)$ & 0.022283 & 0.022788 & 0.024869 & 0.008025 \\
\hline $\mathrm{P}(3)$ & 0.02226 & 0.022561 & 0.022502 & 0.002952 \\
\hline $\mathrm{P}(4)$ & 0.022238 & 0.022336 & 0.020361 & 0.001086 \\
\hline $\mathrm{P}(5)$ & 0.022216 & 0.022114 & 0.018423 & 0.0004 \\
\hline $\mathrm{P}(6)$ & 0.022194 & 0.021894 & 0.01667 & 0.000147 \\
\hline $\mathrm{P}(7)$ & 0.022172 & 0.021676 & 0.015084 & $5.41 \mathrm{E}-05$ \\
\hline $\mathrm{P}(8)$ & 0.022149 & 0.02146 & 0.013648 & $1.99 \mathrm{E}-05$ \\
\hline $\mathrm{P}(9)$ & 0.022127 & 0.021247 & 0.012349 & $7.32 \mathrm{E}-06$ \\
\hline $\mathrm{P}(10)$ & 0.022105 & 0.021036 & 0.011174 & $2.69 \mathrm{E}-06$ \\
\hline $\mathrm{P}(11)$ & 0.022083 & 0.020826 & 0.010111 & $9.9 \mathrm{E}-07$ \\
\hline $\mathrm{P}(12)$ & 0.022061 & 0.020619 & 0.009149 & $3.64 \mathrm{E}-07$ \\
\hline $\mathrm{P}(13)$ & 0.022039 & 0.020414 & 0.008278 & $1.34 \mathrm{E}-07$ \\
\hline $\mathrm{P}(14)$ & 0.022017 & 0.020211 & 0.00749 & $4.93 \mathrm{E}-08$ \\
\hline $\mathrm{P}(15)$ & 0.021995 & 0.02001 & 0.006778 & $1.81 \mathrm{E}-08$ \\
\hline $\mathrm{P}(20)$ & 0.021885 & 0.019034 & 0.004111 & $1.22 \mathrm{E}-10$ \\
\hline $\mathrm{P}(25)$ & 0.021776 & 0.018105 & 0.002493 & $8.24 \mathrm{E}-13$ \\
\hline $\mathrm{P}(30)$ & 0.021667 & 0.017222 & 0.001512 & $5.55 \mathrm{E}-15$ \\
\hline $\mathrm{T})$ & \\
\hline
\end{tabular}

Table 2: Probabilities of Target Nucleus Energy States at

Various Zetas

\section{Acknowledgements}

This work was carried out in MeiL Faculty of Warsaw University of Technology, Poland. The author discloses no competing interest.

\section{References}

1. Kolbe, E., Langanke, K., and Fuller, G.M., (2004). Neutrino-Induced Fission of Neutron-Rich Nuclei. Physical Review Letters. 92(11)

DOI: 10.1103/PhysRevLett.92.111101

2. Kelić, A., Zinner, N., Kolbe, E., Langanke, K., Schmidt, K.-H. (2005). "Cross sections and fragment distributions from neutrino-induced fission on r-process nuclei". Physics Letters B. 616 (1-2): 48-58.

https://doi.org/10.1016\%2Fj.physletb.2005.04.074 
3. Balantekin, A.B., and Fuller, G.M., (2003). Supernova neutrino-nucleus astrophysics. Journal of Physics G: Nuclear and Particle Physics. 29(11).

https://doi.org/10.1088/0954-3899/29/11/007

4. Fuller, G.M., and Meyer, B.S., (1995). Neutrino Capture and Supernova Nucleosynthesis. Astrophys. J. 453(792-809)

5. Qian, Y.Z., (2002). Neutrino-Induced Fission and r-Process Nucleosynthesis. Astrophys. J. 569 (L103-L106). Doi: 10.1086/340643

6. Vannucci. F. (2017). Interactions of neutrinos with matter. Prog.Part.Nucl.Phys. 95 (2017) 1-47. DOI: 016/j.ppnp.2017.03.003

7. Freedman, D.Z., Schramm, D.N., and Tubbs, D.L., (1997). The Weak Neutral Current and its Effects in Stellar Collapse. Ann. Rev. Nucl. Sci. 27(167-207). https://doi.org/10.1146/annurev.ns.27.120177.001123

8. Formaggio, J.A., and Zeller, G.P., (2012). From eV to EeV: Neutrino Cross Sections across Energy Scales. Rev. Mod. Phys. 84, 1307. DOI:https://doi.org/10.1103/RevModPhys.84.1307

9. Scholberg, K., (2015). Coherent elastic neutrino-nucleus scattering. J. Phys.: Conf. Ser. 606 012010. doi:10.1088/1742-6596/606/1/012010

10. Daniel, Z.F., (1974). Coherent effects of a weak neutral current. Phys. Rev. D 9, 1389. https://doi.org/10.1103/PhysRevD.9.1389

11. Vadim, A.B., and Dmitry, V.N. (2018). Coherency and Incoherency in neutriono-nucleus elastic and inelastic scattering. Phys. Rev. D 98, 053004. https://doi.org/10.1103/PhysRevD.98.053004

12. Wolfenstein, L. (1978). Neutrino oscillations in matter. Phys. Rev. D. 17(9): 2369-2374. https://doi.org/10.1103/PhysRevD.17.2369

13. Loureiro, A., et Al. (2019). Upper Bound of Neutrino Masses from Combined Cosmological Observations and Particle Physics Experiments. Phys. Rev. Lett. 123, 081301. https://doi.org/10.1103/PhysRevLett.123.081301

14. Siemens, P.J., and Jensen, A.S., Elements of Nuclei, (Addison-Wesley Publishers, Redwood City, 1987) 